\title{
LA REGENERACIÓN DE LA ASTRONOMÍA Y LA METEOROLOGÍA ESPAÑOLAS: AUGUSTO ARCIMÍS (1844-1910) Y EL INSTITUCIONISMO
}

\author{
Aitor Anduaga Egaña \\ Academic Visitor. Modern History Faculty. University of Oxford (U.K.)
}

\section{RESUMEN}

El artículo trata sobre los intentos por regenerar la astronomía y la meteorología españolas durante el último cuarto del siglo XIX a través de la trayectoria científica y humana del astrónomo amateur y primer meteorólogo profesional español Augusto Arcimís (1844-1910). Se estudia el papel de personalidades vinculadas a la Institución Libre de Enseñanza en la creación del Instituto Central Meteorológico en 1887 y en la difusión de teorías astronómicas modernas.

PALABRAS CLAVE: España, siglo XIX, astronomía, meteorología, Institución Libre de Enseñanza, institucionismo, regeneracionismo, Augusto Arcimís Wehrle, Francisco Giner de los Ríos.

\section{SUMMARY}

The article is about the attempts for regenerating Spanish astronomy and meteorology in the last quarter of the twentieth century through the human and scientific trajectory of Augusto Arcimís (1844-1910), amateur astronomer and the first Spanish professional meteorologist. The role of personalities linked to the Institución Libre de Enseñanza in the creation of the Instituto Central Meteorológico in 1887 and the spread of modern astronomical theories is analyzed.

KEY WORDS: Spain, nineteenth century, astronomy, meteorology, Institución Libre de Enseñanza, institucionismo, regeneracionismo, Augusto Arcimís Wehrle, Fancisco Giner de los Ríos. 
LA REGENERACIÓN DE LA ASTRONOMÍA Y LA METEOROLOGÍA ESPAÑOLAS: AUGUSTO ARCIMÍS (1844-1910) Y EL INSTITUCIONISMO.

Con algunas salvedades, podría afirmarse que, además del 98 literario, se produjo en España otro 98 científico, cimentado sobre el positivismo y fraguado dentro del movimiento regeneracionista que se inició con la entrada de la Restauración borbónica en 1874. La convergencia de un conjunto de fuerzas sociales e intelectuales condujo a una regeneración moral y ética en el dominio político, pero también a una regeneración científica e industrial que acarreó un mayor bienestar social y económico. En el terreno de la astronomía y la meteorología, la regeneración se reviste de matices propios. No sería razonable sobreestimar la influencia de la Institución Libre de Enseñanza en este proceso de renovación, pero tampoco lo sería subestimar su importancia. Puesto que, como acaeció en otros campos de la ciencia - historia natural, geografía, geología, matemáticas-, los institucionistas contrapusieron el libre pensamiento y la renovación a las concepciones dogmáticas y a las inercias del pasado ${ }^{1}$.

Evidentemente no podría insinuarse que la astronomía y la meteorología españolas fueron terrenos de cultivo estériles durante la Restauración ${ }^{2}$. Figuras de la talla de, por nombrar algunos, Antonio Aguilar, Manuel Rico Sínobas, Miguel Merino, Vicente Ventosa y Cecilio Pujazón, e instituciones de solera como los Observatorios Astronómicos de Madrid ${ }^{3}$ y San Fernando, ${ }^{4}$ gozaban de alto crédito, si bien en muchos casos se sustentaba más de la luz que proyectaban pasados gloriosos que de la sombra que arrojaban presentes inciertos ${ }^{5}$. Lejos de defender por tanto, que en el último cuarto del siglo XIX se inició una nueva práctica científica, en el presente artículo trataremos de demostrar que se emprendió la regeneración de unas ciencias cuya situación

1 García CAmARERo, E. (2000), «La regeneración científica en la España del cambio de siglo», Revista de Hispanismo Filosófico, 5, pp. 17-42.

2 Anduaga, A. (2001), La institucionalización y enseñanza de la Meteorología y la Geofísica en España, 1800-1950, Tesis doctoral inédita realizada bajo la dirección de José Llombart Palet, Universidad del País Vasco.

3 Asociación de Amigos del ObServatorio Astronómico de MAdrid, eds. (1992), Doscientos años del Observatorio Astronómico de Madrid, Madrid, Estudio Gráfico Madrid.

4 GonzÁlez, F. J. (1993), Cecilio Pujazón y el Observatorio de la Marina, Cádiz, Servicio de Publicaciones de la Armada.

5 Ten, A.; Moya, T. (1988), «La formación de un astrónomo en la España del siglo XIX», en: Estudios sobre Historia de la Ciencia y de la Técnica, IV Congreso de la Sociedad Española de Historia de las Ciencias y de las Técnicas, Valladolid, Junta de Castilla y León, 1988, pp. 469-484. 
era muy delicada. La propia circunstancia de que durante este periodo se promovieran un mayor número de iniciativas - el Instituto Central Meteorológico (1887), los estudios de astronomía física y de dinámica de la atmósfera, el servicio de predicción del tiempo, la formación de una pléyade de jóvenes astrónomos, por citar algunas - que las que habían sido impulsadas hasta entonces, nos induce a explorar esta avenida.

A continuación tratamos de profundizar una particular visión sobre la mencionada regeneración a través de la trayectoria científica y humana de Augusto Arcimís Wehrle (1844-1910). De espíritu ardiente y talante dinámico, Arcimís se sintió arrastrado, al igual que muchos institucionistas contemporáneos suyos, por el entusiasmo de Francisco Giner de los Ríos — catador y domador de espíritus rebeldes-. Gracias a él y al círculo de amistades influyentes que tejió por toda Europa, lideró primero desde su pequeño observatorio de Cádiz, y luego, desde la Institución Libre de Enseñanza, una paulatina renovación que culminó con la creación del Instituto Central Meteorológico, en 1887.

Tanto en sus planteamientos pioneros como en su empecinamiento contra la ciencia oficial, Arcimís no estaba saldando simplemente una deuda intelectual personal. Más bien, se estaba esforzando por ensanchar los horizontes de la astronomía y la meteorología españolas. Esta tarea iba a dejar una huella imborrable en toda una generación de jóvenes astrónomos cuyas inquietudes autodidactas recordaban la pasión del joven Arcimís por la regeneración científica en una época de gran revuelo social y cultural que acompañó a la instauración de la Restauración borbónica.

El joven Arcimís: entre la astronomía amateur y la Institución Libre de Enseñanza

El carácter entusiasta de Arcimís y la variada gama de campos de saber que cultivó fueron extraordinarios. Nacido en Sevilla en 1844 en el seno de una familia acomodada, pero trasladado a Cádiz a la edad de cuatro años, era hijo de un comerciante de origen vasco-francés. ${ }^{6}$ En Cádiz, ciudad mesocrática, en aquella época con un talante naval y comercial, aunque también, pero en menor

6 Se tienen noticias de él a través de varias fuentes: Nicolás Sama, Ayudante de Arcimís en el ICM, esboza su biografía en los Anales de la Sociedad Española de Meteorología, 1927, 1 (2), pp. 3-4; José Jiménez-Landí hizo otro tanto en La Institución Libre de Enseñanza, II. Período parauniversitario, Taurus, Madrid, 1987, p. 613; y también puede consultarse la Enciclopedia Universal Ilustrada Europeo-Americana, Madrid, Espasa-Calpe, 1958, V, p. 1297. 
medida, industrial, la inclinación de sus padres se centró en la consecución de una formación elitista y severa, que le permitiera acceder a los estudios con más porvenir económico (Farmacia, Medicina) a la vez que administrar los negocios familiares ${ }^{7}$. Ingresó en el distinguido Colegio marianista de San Felipe Neri, núcleo molecular de la ciudad, donde se respiraba un ambiente de rigurosa disciplina que, contrariamente a lo esperado, no iba a influir en su futuro a la hora de aplicar este régimen en las organizaciones que tendría a su cargo ${ }^{8}$. Aquí recibió una notable formación de idiomas, entre ellos el francés, inglés e italiano, gracias a los métodos de enseñanza de H. G. Ollendorf, que habían sido adaptados por el profesor Eduardo Benot ${ }^{9}$. Por entonces, la fisonomía de Cádiz era tan marianista como podía ser mercantil o burguesa. Tras concluir el bachillerato, inició los estudios en la Facultad de Farmacia, que había sido recientemente inaugurada a la sombra de la célebre Facultad de Medicina, obteniendo el doctorado, aunque nunca llegaría a ejercer como farmacéutico.

Pese a que la formación marianista, estricta, fue para muchos un estilo de pensamiento, de vida, en Arcimís supuso, en cambio, un apremio por alcanzar la libertad y la neutralidad religiosa, una confianza ciega en la educación liberal, integral y armónica. Las siguientes palabras, escritas por el meteorólogo Nicolás Sama, condensan mucho de lo que a propósito de su personalidad querríamos reflejar en tal sentido:

Fue Arcimís atildado y pulcro, enérgico y luchador, de convicciones avanzadas, y extraordinariamente comprensivo y tolerante, de modales aristocráticos y de espíritu finamente cultivado; matizaba su siempre amena e instructiva conversación con fino gracejo andaluz. Amante de la naturaleza y experto marino, pasaba sus ratos de ocio en el campo o en el mar. Enemigo de vanidades externas, rechazó toda clase de condecoraciones ${ }^{10}$.

Fue, probablemente, su fe vívida en los beneficios del librepensamiento y del positivismo científico, lo que propició que se dedicara en los próximos años a viajar, presumiblemente porque las condiciones económicas de su familia se lo permitieron. Recorrió Europa, visitando destacados observatorios

7 Ramos Santana, A. (1993), Cádiz en el siglo XIX. De ciudad soberana a capital de provincia, Cádiz, Silex.

8 GonzÁlez, J. (1983), El Colegio de San Felipe Neri, Cádiz, Caja de Ahorros, p. 106.

9 BENOT, E. (1856), Método del Dr. Ollendorff para aprender a leer, hablar y escribir un idioma cualquiera adaptado al francés: para uso de los alumnos del Colegio de S. Felipe Neri de Cádiz, Cádiz, Imprenta y Librería de la Revista Médica.

10 SAMA (1927), p. 3. 
en Francia, Alemania e Inglaterra, y vivió en Londres durante un tiempo. Pocos detalles de las amistades que entabló en torno a estas visitas han sobrevivido. Pero parece seguro que el periplo contribuyó, por lo menos, a acrecentar su interés por la astronomía y por la meteorología. Su fascinación por las ciencias de observación se enraizaba enteramente en la tradición astronómica gaditana y en el estilo autodidacta que habían mostrado algunos profesores andaluces en la década de $1870^{11}$. El cielo andaluz era un marco especialmente privilegiado para el estudio, cuya atmósfera reunía las condiciones de pureza y diafanidad requeridas ${ }^{12}$, características que justificaban tanto la actividad científica, durante siglos, del Observatorio de San Fernando, como las observaciones efectuadas, durante el año 1870 , por los catedráticos del Instituto Provincial de Cádiz con motivo del eclipse de Sol. En éste, su novedad más significativa estribaba no tanto en las mediciones de las protuberancias solares, de la corona o de la irradiación solar, sino en el proyecto de observaciones espectroscópicas que, de haberse llevado a cabo, hubiera supuesto, con toda probabilidad, la primera aplicación en España de la técnica espectroscópica a la astronomía ${ }^{13}$. El minucioso programa pretendía, además, averiguar la posible influencia del eclipse en los idiotas y en los dementes, para lo cual se contó con la colaboración de los médicos del Hospital del Carmen y de la Casa de Dementes.

En este contexto, no es sorprendente que Arcimís hallara en Cádiz un clima propicio para la práctica de la astronomía. Su estancia en el extranjero le había permitido dominar fluidamente varios idiomas, granjear amistades provechosas con astrónomos prestigiosos e imbuirse de un liberalismo y positivismo románticos que respondían a los adelantos de esos países y a la penuria de España. Estas primeras inquietudes se vieron parcialmente colmadas cuando, a su regreso, instaló en su casa un pequeño observatorio astronómico y meteorológico, conocido familiarmente como la Specola, que estaba equipado, entre otros instrumentos, con una ecuatorial y con aparatos registradores. No obstante, fue la estrecha amistad que entabló con Francisco Giner de los

11 Simo, P. (1974), Memoria leída en el acto de doctorado. Fenómenos observados durante el eclipse total de Sol, Jerez, Martín Díaz. Véase también Memoria sobre el eclipse total de sol visible en Málaga el 22 de Diciembre de 1870, El Avisador Malagueño, Málaga, 1871.

12 X. X. (1858), «Astronomía», Revista Jerezana, 1, 4.

13 Rubio, V.; Fdez. Fontecha, Fco.; Alcolea, J. (1871), Eclipse total de sol del 22 de diciembre de 1870: memoria de las observaciones verificadas por varios catedráticos del Instituto de Cádiz, unidos a otras personas cientificas de esta capital, Cádiz, Imprenta de la Revista Médica, p. 10. 
Ríos, iniciada a mediados de 1875 y que se prolongaría hasta su fallecimiento, en 1910, lo que marcó terminantemente su trayectoria científica y humana. ${ }^{14}$ Las circunstancias trágicas que motivaron su encuentro guardan cierta semejanza: Giner había sido confinado, con motivo de las disposiciones del Ministro Manuel Orovio contra la libertad de enseñanza, al Castillo de Santa Catalina en Cádiz, mientras que Arcimís y su esposa Elodia estaban atravesando una delicada situación económica en el negocio familiar de vinos que regentaban. A esta penuria laboral se sumó la desdicha humana con la pérdida de tres de sus hijos, entre 1875 y 1878, a lo que Giner respondió con muestras sinceras de apoyo y solidaridad. Todo ello acentuó, sin duda, la estima y la admiración que Arcimís sentía hacia él, por su personalidad, por su pensamiento, «por su exaltado fervor por cuanto pudiese contribuir a la dignidad del hombre y a la renovación intelectual, moral y científica de España» ${ }^{15}$.

El vigor que transmitía Giner recibió, entre tantas desventuras personales, una acogida cálida por parte de la familia, en parte por el carácter enfermizo de Elodia, pero también por la naturaleza de las propias aspiraciones de Arcimís, que guardaban mayor relación con la libertad de pensamiento, la emancipación del ser humano, y sobre todo, con la regeneración de la astronomía y la meteorología españolas. En mayor o menor grado, Giner contagió su ímpetu y optimismo a los amigos de Arcimís, José Macpherson y Alejandro San Martín, a quienes les unía el firme afán de reformar el panorama científico y cultural español de finales del siglo XIX. «Debían de ser, sin duda, extraordinarias la capacidad de entusiasmo y el atractivo personal que tenía don Francisco cuando podía incluso hacer cambiar el rumbo de una vida» ${ }^{16}$; fascinación que, en palabras de A. Jiménez-Landi, era una auténtica ósmosis espiritual, y que alcanzaba cotas máximas según se desprende del párrafo que le dedicó Arcimís en una de sus cartas:

Yo creo que quiero a $\mathrm{Vd}$. tanto que no me inspira ni respeto, ni temor, ni me guardo más de $\mathrm{Vd}$. que de mi sombra, y cuando muchas veces me censura Vd. jamás se me ocurre que es mi amigo, ni mi padre, ni mi preceptor el que me reprende, sino

14 Fruto de esta relación, se conserva una correspondencia de más de 125 cartas en la Biblioteca de la Real Academia de la Historia de Madrid, Fondo Giner de los Ríos. El historiador Luis García de Valdeavellano, nieto de Arcimís, resumió parte de la correspondencia en una conferencia que celebró en la Corporación de Antiguos Alumnos de la Institución Libre de Enseñanza, el 24 de abril de 1980, sobre el tema Mi abuelo Augusto Arcimís y su correspondencia con don Francisco. El Instituto Central de Meteorología.

15 García de VALDEAVELlano (1980), p. 4.

16 García de Valdeavellano (1980), p. 5. 
la voz imperiosa de mi propia conciencia, que a despecho mío he de escuchar. ¡Bendito sea mil veces Orovio, si es que a él le debo el haber conocido a Vd.!17

DEL REGENERACIONISMO DE LA ASTRONOMÍA A LA PROFESIONALIZACIÓN DE LA METEOROLOGÍA

Así como las primeras correspondencias de Arcimís ilustran claramente su deseo por ensanchar sus horizontes culturales e imitar el comportamiento ético de Giner, de la misma manera este proceso de seducción éticointelectual debe ser enmarcado en el contexto de una idea más general como era la de rebelarse ante las prácticas de los astrónomos de los observatorios oficiales, en su opinión más inmersos en las intrigas del poder que en la consecución de méritos científicos. Fue en este entorno en el que Arcimís llevó a cabo una intensa actividad astronómica a partir de 1874. Su táctica estuvo encaminada, en primer lugar, a trabar amistades con reconocidos astrónomos de talla internacional, como fueron los Padres Angelo Secchi y F. Denza, Urbain Le Verrier, M. William Huggins, Browning, etc., y, seguidamente, a integrarse en círculos académicos prestigiosos, como en la Royal Astronomical Society of London, en diciembre de 1875, y en la Società degli Spettroscopisti Italiana, en 1874, para desde esta posición, iniciar una fecunda labor de publicaciones en revistas extranjeras acreditadas que le permitiera rivalizar con la astronomía oficial española, en su deseo de «hacer una guerra a muerte» a los Observatorios de San Fernando y Madrid. A este respecto, su actitud fue la propia del grupo de jóvenes institucionistas para los cuáles Europa era la luz que alumbraba el camino a seguir entre la niebla de atraso y oscuridad españolas en una época en la que las demandas de renovación e industrialización adquirían fuerte protagonismo. Una lectura de la correspondencia de Arcimís con Giner muestra explícitamente cuáles fueron los grandes temas: aquí Europa, liberalismo, regeneración astronómica y meteorológica, y modernización industrial se acoplaban perfectamente al unísono. En palabras del propio Arcimís, los responsables de la astronomía oficial, en clara alusión a Miguel Merino y Cecilio Pujazón, eran parte del problema:

No deja de ser triste que todas las cosas que puedan adentrarme en mis trabajos y estimularme a continuar hayan de venir del extranjero: instrumentos, libros, periódicos y recompensas. Todo ha de ser de otras tierras que de esta desgraciada España y mi propio idioma para bien poco puede servirme y mis relaciones con los

17 Carta de A. Arcimís a F. Giner de los Ríos del 13 de noviembre de 1876. 
científicos de aquí, para menos todavía. ¿Pero habrá aquí hombres científicos? Casi puede creerse que no y no me parece muy atrevido decir que los sabios oficiales han obtenido sus puestos por asalto más que por sus méritos. Si se les conoce es por los puestos que ocupan y no por sus méritos personales; ¿se puede saber qué debe la ciencia a los directores de los Observatorios de San Fernando y Madrid? ${ }^{18}$

En sus contribuciones científicas, Arcimís no fue un liberal doctrinario ni un agitador crítico sino un observador amateur de planteamientos pioneros y avanzados. De hecho, en fecha tan temprana como 1874, publicó varios trabajos en el órgano de la Società degli Spettroscopisti Italiani, una revista que había comenzado a editarse a partir de 1872, convirtiéndose en la primera publicación especializada de astrofísica en la historia de la astronomía moderna ${ }^{19}$. El rigor con que efectuaba sus observaciones fue suficiente para que su nombre adquiriera gran crédito entre el círculo de astrónomos italianos. En Italia, dicha sociedad representaba la primera tentativa de coordinación de la investigación espectroscópica aplicada a la física solar ${ }^{20}$; y en España, Arcimís se convertía en el abanderado por antonomasia de la astrofísica.

Arcimís adoptó en sus primeras publicaciones científicas una posición que resultaría incómoda para muchos astrónomos oficiales españoles. Se basaba en el argumento de que la técnica espectroscópica era esencial para comprender la composición química de las estrellas y su ulterior catalogación de acuerdo a los espectros luminosos ${ }^{21}$. Esta postura resuelta, aunque un tanto presuntuosa, por proceder de un amateur, podría haber herido el orgullo profesional de aquéllos por cuanto que apuntaba a la espectroscopia estelar como una técnica revolucionaria de la astronomía física que no había sido aplicada aún en España ${ }^{22}$. Ciertamente, cuando Arcimís envió una carta al Padre Secchi dando cuenta de sus observaciones espectroscópicas de estrellas fugaces, hacía hincapié en su

18 Carta de A. Arcimís a F. Giner de los Ríos del 18 de septiembre de 1875.

19 ARCIMís, A. (1874), «Studi spettroscopici delle Stelle cadenti fatti dal signor Arcimis a Cadice. (Lettera diretta al P.A. Secchi)», Memorie della Societá degli Spettroscopisti Italiani, 3, 100-101; ARCIMís, A. (1875), «Observazioni sulla luce zodiacale fatte in (Cadice) Spagna nel 1875», Memorie della Societá degli Spettroscopisti Italiani, 4, 33-37.

20 Chinnici, I.; Gramatowski, W. (2001), «Le carte di Angelo Secchi, S. J.», Nuncius, 16, p. 579.

21 Años más tarde, haría extensible esta técnica a la predicción del tiempo. Véase: ARCIMís, A. (1984), «El espectroscopio y la previsión del tiempo», Boletín de la Institución Libre de Enseñanza, 8, 37-39; 53-55.

22 Para una discusión sobre los espectros estelares y su relación con el desarrollo de la astrofísica, véase: HEARnshaw, J. B. (1986), The Analysis of Starlight: One hundred and fifty years of Astronomical Spectroscopy, Cambridge, Cambridge Univ. Press, pp. 208-254. 
condición de outsider y en sus modestos medios; y se mostraba extremadamente halagado cuando Secchi describía su sencilla Specola como el Observatorio de $\mathrm{Cádiz}^{23}$. Del mismo modo, no ocultaba su alegría cuando el Padre Denza reseñaba sus observaciones en varias revistas italianas, editadas por los Observatorios de Moncaleri y de Turín, al lado de astrónomos tan ilustres como Sir Joseph Norman Lockyer y Lorenzo Respighi; y Arcimís le recordaba con ingenio andaluz que «no era un astrónomo sino un desgraciado que alguna vez para estirar los músculos cervicales alzaba la cabeza al cielo» ${ }^{24}$.

Como se ha aludido anteriormente, la experiencia de Arcimís, que se nutrió en gran parte de lo que había observado en sus viajes por Europa, fue clave en su concepción astronómica. Para sus objetivos de regeneración, las relaciones que había entablado con notables especialistas de la astronomía física, como eran M. W. Huggins, autor del tratado Analyse spectrale des corps celestes, de 1866, el aristócrata Lord J. L. Lindsay, fundador de un Observatorio que llevaba su nombre en Aberdeen y autor de la obra Astronomy, de 1878, y Sir T. Maclear, un médico de profesión que se convirtió en astrónomo real y director del Royal Cape Observatory, en Sudáfrica, fueron determinantes en su puesta al día científica de las ciencias de observación, paso previo para la europeización de España. Una de las frases de su epistolario con Giner, «yo aguardo como aquél la última moda para hacerme mi vestido intelectual», sintetiza poderosamente su capacidad de improvisación y de perfeccionamiento en el bullir científico de aquellos años finiseculares. No obstante, parece muy probable que ya se hubiera familiarizado con la técnica espectroscópica en la carrera de Farmacia y con la forma de efectuar con éxito los análisis espectrales ${ }^{25}$. Su destreza en el manejo del espectroscopio quedó patente en las prácticas de espectroscopia con sangre humana que realizó en 1876 en colaboración con San Martín, quien confesaba, con sorpresa, no haber visto nunca un espectro ${ }^{26}$. La adaptación y efectividad del nuevo procedimiento eran

23 Carta de A. Secchi a A. Arcimís, 1873/76. Archivo della Pontificia Università Gregoriana, Roma.

24 Carta de A. Arcimís a F. Giner de los Ríos del 1 de abril de 1876.

25 El uso de la espectroscopia como método de análisis médico no era ajeno en las Facultades de Farmacia y Medicina. Véanse los discursos de doctorado: FAlCES DE OdiagA, E. (1877), Análisis espectral: discurso leído por el Licenciad en Farmacia..., Madrid, Imp. Est. y Galv. Aribau; CEBrián, E. (1883), Del análisis espectral en medicina [Manuscrito]. Tesis de doctorado leida en la Universidad Central el 21 de junio de 1883, Madrid.

26 Carta de A. Arcimís a F. Giner de los Ríos del 13 de mayo de 1876. Sobre la figura de A. San Martín, véase: Palma Rodriguez, F. (1997), Vida y obra del doctor Alejandro San Martín Satrústegui, San Sebastián, Sociedad Vasca de Historia de la Medicina. 
inauditos, marcando, como intuyó Arcimís, un momento de ruptura en la instrucción tradicional de la astronomía descriptiva o de posición, a cuya práctica se habían adherido fervientemente los directores de los Observatorios oficiales y quienes, a buen seguro, habrían preferido ser los precursores de la espectroscopia estelar en vez de meros espectadores 27 .

Al igual que había sucedido en Italia, la seriedad y la madurez de los trabajos de Arcimís fueron suficientes para que su obra fuera acogida favorablemente en Inglaterra. Aquí había ganado una gran reputación por su nombramiento como Fellow de la Royal Astronomical Society of London. En su relato a Giner de dicha distinción, Arcimís elogiaba las excelencias de un país en el que se apreciaban los esfuerzos por difundir el gusto a la astronomía aunque fueran realizados, como en su caso, con medios escasos. En su opinión, la amplia cultura de organización y libertad de las instituciones británicas alimentaba un espíritu de iniciativas científicas que no tenía parangón en España y potenciaba la aparición de revistas especializadas como vehículos de difusión de la ciencia. Sus estudios sobre los eclipses de los satélites de Júpiter y el disco de Venus se publicaron en el Monthly Notices of the Royal Astronomical Society, órgano de la Sociedad de la que era miembro, dando motivo a notables discusiones $^{28}$; sus observaciones acerca de las ocultaciones de las estrellas efectuadas con el telescopio ecuatorial de Browning se noticiaron en The Observatory, la revista mensual del Observatorio de Greenwich ${ }^{29}$; crónicas científicas suyas vieron la luz en la revista Nature ${ }^{30}$; y también sus trabajos sobre los eclipses lunares y solares se valoraron en los círculos académicos ${ }^{31}$. Fue en Inglaterra y

27 La nueva astronomía física se difundió a través de círculos académicos ajenos a la astronomía oficial. Véase, por ejemplo, LANDERER, J.J. (1889), Introducción á la astronomía física, Barcelona, Redacción y administración de la "Crónica científica»; y CASTELLS, P. (1897), Fundamentos de espectroscopia celeste. Discurso de doctorado leido en la Universidad Central, Madrid, Est. Tip. de la viuda e hijos de Tello.

28 ARcimís, A. (1876), «Phenomena of Jupiter's satellites observed at Cadiz», Monthly Notices of the Royal Astronomical Society, 37, 259-260; ARCIMís, A. (1876), "On the visibility of the unilluminated portion of the disc of Venus», Monthly Notices of the Royal Astronomical Society, 37, 259.

29 ARcimís, A. (1879), «The occultation of Antares, 1879, July 28», The Observatory, 3, 148; Arcimís, A. (1879), "Conjunction of Mars and Saturn, 1879, June 30, observed at Cádiz», The Observatory, 3, 107-108.

30 Véase, por ejemplo, ARcimís, A. (1879), «Earthquake at Cadiz», Nature, 19, 555, (3 April 1879); y "Arcimis' observations of Sunset Phenomena in Spain», Nature, 30, 1884, 324.

31 ARCimís, A. (1876), "Observations of the lunar eclipse, 1876 September 3, made at Cadiz», Monthly Notices of the Royal Astronomical Society, 37, 12-13; ARCIMís, A. (1877), «Lunar eclipse of 1877 February 27, observed at Cadiz», Monthly Notices of the Royal Astro- 
en Italia, precisamente, donde mayor eco alcanzó su más destacada contribución concerniente al análisis espectral de la luz zodiacal, trabajo que realizó con la ayuda de un espectroscopio, modelo Hoffmann, de cinco prismas, y que redundó en una discusión con el profesor Wright del Yale College $e^{32}$.

En contraste, la atención que se prestó en Francia a sus observaciones no fue la que él esperaba. El país del que Arcimís había declarado ser su predilecto y que había conocido íntimamente a través de su familia tenía poco en común con el ambiente que él percibió en los observatorios y círculos académicos, tras su visita a París en 1888. Una parte de culpa residía en la animadversión que sentía hacia el astrónomo francés Camile Flammarion y todo lo que éste representaba. La proliferación en España de las «sociedades espirituales llamadas de Flammarion» infligía, según él, un enorme daño a la ciencia en general y a la astronomía en particular ${ }^{33}$. La reacción de aquél, como se observa en el anuario del órgano de la Société Astronomique de France de 1892, fue contundente pero sibilina, cuando mencionaba a Comas, Fontserè y Landerer como los únicos científicos españoles que se distinguían por las observaciones solares.

No decayó el interés de Arcimís por el conocimiento innovador en sus arremetidas contra la astronomía oficial, sobre todo a través de sus correspondencias y artículos en publicaciones extranjeras, pese a la cada vez más inquietante situación económica de su negocio de vinos. El relato de la búsqueda de Vulcano, un supuesto planeta que jamás se descubrió, y la correspondencia que sostuvo con U. Leverrier, director del Observatorio de París, a cuenta de dicho motivo, nos revelan a Arcimís lleno de un entusiasmo teñido de inquietud. Por un lado, las cartas del astrónomo francés durante 1877 le servían de estímulo en la tarea que se había impuesto a sí mismo como celador e iniciador del regeneracionismo astronómico. Pero por otro lado, se acrecentaba su temor de que España había quedado científicamente rezagada de los países europeos del mismo entorno cultural y de que si la astronomía española quería competir en observaciones, tanto en cantidad como en calidad,

nomical Society, 37, 400; ARCiMís, A. (1877), «Observations of occultations of stars by the Moon, made at Cadiz in the year 1876», Monthly Notices of the Royal Astronomical Society, 37, 261; ARCimís, A. (1879), «The eclipse of 1879, July 18», The Observatory, 3, 126; 250.

32 Arcimís, A. (1875), «Observations of the zodiacal light at Cadiz», Monthly Notices of the Royal Astronomical Society, 36, 48-51. Previamente, el estudio había sido publicado en el Bulletino Meteorologico del Collegio Romano, que era dirigido por el Padre A. Secchi.

33 Carta de A. Arcimís a F. Giner de los Ríos del 7 de mayo de 1883. 
comparables a las que el propio Arcimís había estado realizando, era necesaria una vasta red de observadores amateurs ${ }^{34}$.

En este aspecto la llegada a Cádiz de Augusto González de Linares y Manuel Bartolomé Cossío, que junto a San Martín y a J. Machperson conformarían un grupo de aficionados a la astronomía ${ }^{35}$, le infundía un nuevo brío en su particular lucha por sus ideales más perseguidos - la renovación científica y la transformación socio-cultural de la sociedad española- precisamente en un momento en que los proyectos astronómicos de Arcimís - los espectros de las estrellas variables, la luz zodiacal, las estrellas fugaces, las ocultaciones y eclipses de los satélites planetarios, las estrellas binarias y múltiples- se veían alentados con su reciente, aunque infundado, descubrimiento de Vulcano. Arcimís, así se lo confesaba a Giner, se sentía el «fundador de la astronomía gaditana ${ }^{36}$. E iba más allá, al declarar que su sueño era formar y reclutar una «pléyade de astrónomos modestos», una generación de espíritus combativos que, como él, fuera una pieza clave para la anhelada regeneración y para la batalla contra la ciencia oficial.

En una época de acrecentamiento en los años 70 de las dificultades económicas en sus negocios y de su progresivo alejamiento de la iglesia cristiana, decide traducir la obra de John W. Draper, la Historia de los conflictos entre la religión y la ciencia ${ }^{37}$. Sin duda, Arcimís encontró sumamente intrigante el hecho de que se tratara de una historia de la ciencia efectuada desde la óptica de los condicionamientos sociales e ideológico-religiosos del autor, y de que representara un serio alegato en contra de la constitución dogmática de la fe católica. A este respecto, los consejos de Giner le sirvieron para que Nicolás Salmerón se hiciera cargo del prólogo, de manera brillante según el propio Draper, que elogió sus esfuerzos «en pro de la causa de las ideas liberales» ${ }^{38}$.

34 Para una visión general de la astronomía amateur en España, véase: OLIVER, J. Ma (1997), Historia de la astronomía amateur en España, Madrid, Equipo Sirius, D.L. Sobre la organización de la astronomía en Gran Bretaña, véase: CHAPMAN, A. (1998), The Victorian amateur astronomer: independent astronomical research in Britain, 1820-1920, Chichester, J. Wiley.

35 Véase, por ejemplo, la conferencia pronunciada en el curso académico 1877-78 en la Institución Libre de Enseñanza por GonZÁlEZ DE LiNARES, A. (1877), La vida de los astros, Madrid, Conde y Cia.

36 Carta de A. Arcimís a F. Giner de los Ríos del 8 de enero de 1878.

37 Draper, J. W. (1876), Historia de los conflictos entre la Religión y la Ciencia. Traducción directa del inglés por Augusto T. Arcimís, de la Real Sociedad Astronómica de Londres. Con un prólogo de Nicolás Salmerón, Madrid, Impr. de Aribau y Ca . La traducción de Arcimís se volvió a editar en 1885, 1886 y 1888. La versión original de la obra data de 1873 .

38 Carta de J. W. Draper a A. Arcimís del 17 de febrero de 1876. 
Salmerón subrayaba que la rémora del pasado continuaba aún hipotecando la libre actividad intelectual en el presente, una actividad que seguía siendo una meta a conquistar en España. Por eso, es muy probable que Arcimís coincidiera con la afirmación de éste de que el principal mérito de la obra era que tendía a «consagrar la libertad de pensamiento» y que su éxito en España serviría «eficazmente para sacudir el letargo en que yace la conciencia religiosa y científica» ${ }^{39}$.

Pese a que la traducción no reportó pingues beneficios económicos a Arcimís, sí que le proporcionó notoriedad, e hizo que su nombre se viera incluido entre el elenco de intelectuales etiquetados con el ambiguo epíteto de heterodoxos. A esto contribuyó, indudablemente, el eco de la obra de Draper, que tuvo en España numerosas réplicas y de considerable altura doctrinal, tanto por parte de los librepensadores como por parte de la apologética católica ${ }^{40}$. En este aspecto, el propio prólogo de Salmerón, que aporta una convincente prueba de cómo el conflicto genérico entre ciencia y religión se imbrica en España, suscitó una contundente respuesta de Menéndez Pelayo, generándose así uno de los debates ideológicos claves de la llamada "polémica de la ciencia española». De hecho, la obra de Draper y la teoría de Darwin fueron «los dos blancos primordiales» de la apologética española de la época ${ }^{41}$. En vista de esto, no es sorprendente que Arcimís declarara eufórico que aún era posible una regeneración en España ya que todavía no se había extinguido «la visualidad ni el fuego sagrado de la ciencia» ${ }^{42}$.

En los tratados clásicos de la segunda mitad del siglo XIX se podía observar claramente la voluntad de convertir a la astronomía en un saber enciclopédico, casi en un objeto de culto, donde las ciencias físico-matemáticas se distinguían como modelo de cientificidad y donde la fe del progreso como nueva religión era reivindicada junto a las consignas de modernización y de la filosofía positiva. Estas actitudes románticas, de recopilación del conocimiento astronómico, que afloraban en las obras de Secchi, Lockyer, Proctor y Guillemin, entre otros, jugaron en Arcimís un papel decisivo, de ruptura con la tradición, de paso hacia delante, a la hora de publicar si no la mejor, sí la más productiva y laboriosa de

39 DRAPER (1876), pp. LXI-II.

40 Véase el lúcido prólogo de Diego Núñez sobre la temática de la obra y la reacción de la apologética cristiana, en DRAPER, J. W. (1987), Historia de los conflictos entre la religión y la ciencia, Barcelona, Alfa Fulla.

$41 \quad$ Op. cit. p. 37.

42 Carta de A. Arcimís a F. Giner de los Ríos del 1 de abril de 1876. 
sus obras, El Telescopio Moderno ${ }^{43}$. La falta de originalidad en el contenido era reconocida por el propio autor, que la definía como una exposición popular de los últimos descubrimientos de los grandes sabios ${ }^{44}$. Pese a que la obra era fruto de un encargo, más relacionado con fines lucrativos que intelectuales ${ }^{45}$, pronto sirvió para que los jóvenes astrónomos, como Joseph Comas y Eduard Fontserè, se iniciaran de manera autodidacta en la astronomía de posición ${ }^{46}$. Por el acopio de obras y revistas especializadas de que hizo gala, así como por la abundante información que contenía, puede afirmarse que constituyó una fuente imprescindible actualizada sobre la investigación astronómica internacional ${ }^{47}$.

La actitud pionera de Arcimís se extendió también a la meteorología. El desarrollo del telégrafo había ampliado las expectativas de los meteorólogos al posibilitar la transmisión inmediata de las observaciones meteorológicas, aunque no había repercutido directamente en el avance de la predicción del tiempo. Esta comenzó a desarrollarse cuando los físicos y meteorólogos se percataron de la importancia de los movimientos de las masas de aire en las inclemencias del tiempo ${ }^{48}$. El cambio en la tecnología de las comunicaciones trajo consigo un cambio en la concepción estática que se tenía de la atmósfera. La meteorología dinámica fue tratada, si bien esporádicamente, por los

43 El primer volumen se editó en México (J.F. Parrés y Cía) en 1878 y el segundo en Barcelona (Montaner y Simón) en 1879.

44 LlOMBART, J. (2000), «Ciencia, religión y literatura fantástica: algunas aportaciones al debate sobre la pluralidad de mundos habitados», en ed., M.S. de Mora CHARLES, Los mundos posibles, Donosita-San Sebastián, Publicaciones del Departamento de Filosofía de la UPV/EHU, 2000, pp. 22-53.

45 En principio se trataba de traducir las obras Le Soleil, de A. Secchi; The Moon, de Carpenter; Spectrum Analysis, de Schellen; y The Modern Telescope, de Lockyer, para la casa editorial Montaner y Simón de Barcelona.

46 BARCA, F. X. (1995), «Joseph Comas i Solà: L’Astronomia de posició», en dirs., J. M. Camarasa, A. Roca, Ciència i tècnica als Països Catalans. Una aproximació biogràfica als darrers 150 anys, Barcelona, Fundació Catalana per a la Recerca, pp. 797-798.

47 En 1901, se volvió a editar la obra en Barcelona, pero con un nuevo título, Astronomía popular. Descripción general del cielo. En ésta, a diferencia de la anterior, se mencionaban los trabajos de astrónomos españoles, y en concreto los de Fontserè, Comas y Lánderer, efectuados entre 1890 y 1900.

48 Sobre el estado de la meteorología dinámica en la segunda mitad del siglo XIX, ver: KutzBACH, G. (1979), The thermal theory of cyclones. A history of meteorological thought in the nineteenth century, Boston, American Meteorological Society; DAMIENS, J. (1987), «La météorologie au XIX ${ }^{\mathrm{e}}$ siècle», La Météorologie, 17, 18-29; y FIERRO, A. (1991), Histoire de la Météorologie, Paris, Editions Denoël, p. 214. 
meteorólogos españoles. Autores como Manuel Iranzo Benedito ${ }^{49}$, Josep Ricart i Giralt ${ }^{50}$ y Angel Rodríguez de Prada ${ }^{51}$ repararon en la importancia que el conocimiento del curso de los vientos y las causas de sus desviaciones tenía para la evolución del tiempo. Pero a finales del siglo XIX el desconocimiento de esta circunstancia era casi general en España.

Arcimís, decíamos, extendió su talante pionero a la meteorología moderna, a través de la obra La circulación atmosférica ${ }^{52}$, editada en fecha tan temprana como 1895, y a través de una serie de artículos sobre la meteorología dinámica que se publicaron en el Boletín de la Institución Libre de Enseñan$z a^{53}$, el resultado de varios años de labor académica en dicha institución. Nuevamente, el astrónomo gaditano se adhería enérgicamente a las corrientes teóricas más vanguardistas de la época, implícitamente en la selección de los autores y en el tratamiento matemático de las cuestiones, que se centraban en la importancia de la circulación del aire para la predicción meteorológica. Los estudios de Oberbeck de 1888, sobre las soluciones matemáticas de las ecuaciones del movimiento atmosférico, o de Helmholtz, en 1888 y 1889, postulando que la circulación debía tener en cuenta turbulencias horizontales y verticales que condicionaban el cálculo de los flujos de calor, quedaban extensamente reseñados en la obra La circulación atmosférica ${ }^{54}$. Claramente, con la elección de un tema tan inédito como la circulación de la atmósfera, Arcimís había querido avivar la inquietud de los meteorólogos aficionados y

49 Ver: IrAnZo Benedito, M. (1889), Ensayos de Meteorología Dinámica con relación a la Península Ibérica, Valencia, Imprenta Domenech.

50 Ver: RicART I GIRALT, J. (1892), La Previsión científica del tiempo. Memorias de la Real Academia de Ciencias y Artes de Barcelona, Barcelona, La Academia.

51 Ver: Rodríguez de PradA, A. (1902), Meteorología dinámica. $2^{\mathrm{a}}$ edición, Madrid, Viuda e Hijos de Gómez Fuentenebra; y Rodriguez DE PRADA, A. (1910), «De Meteorología dinámica». En: Asociación Española para el Progreso de las Ciencias. Congreso de Valencia, Madrid, Imprenta de Eduardo Arias, III, pp. 59-64.

52 Arcimís, A. (1895), La circulación atmosférica, Madrid, Ed. Almacén de Papel y Objetos de Escritorio.

53 ARCimís, A. (1888), «La meteorología moderna», Boletín de la Institución Libre de Enseñanza,12, 284-287; 299-300; ARCIMís, A. (1891), «Clasificación de las nubes», Boletín de la Institución Libre de Enseñanza, 15, 348-352. La vocación divulgativa y pedagógica de Arcimís se puso de manifiesto en sus colaboraciones en el B.I.L.E., un total de quince concursos entre 1884 y 1891.

54 Compárense las fuentes bibliográficas de que dispuso Arcimís con los trabajos teóricos citados en el estudio histórico de LORENTZ, E. N. (1983), "A History of Prevailing Ideas about the General Circulation of the Atmosphere», Bulletin of the American Meteorological Society, 64 (7), pp. 730-734. 
dejar constancia de las carencias de los observatorios oficiales. Y a tenor de los resultados, tuvo éxito. Su obra fue alabada en los círculos académicos y en las agrupaciones meteorológicas; y le sirvió de estímulo para que, a comienzos del siglo XX, publicase el tratado de divulgación Meteorología, que gozó de un gran éxito editorial ${ }^{55}$.

Pese a estos motivos de ilusión, Arcimís había sufrido serios quebrantos en los negocios comerciales, que le habían obligado a aceptar un trabajo en una fábrica de alquitrán en Madrid. Fue a través de Giner, una vez más, que Arcimís pasó a desempeñar las cátedras de astronomía y de física en las aulas de la Institución Libre de Enseñanza a partir de 1884. Su ideal instructivo se ajustaba al modelo institucionista de enseñanza activa, en el que no se aspiraba a imponer conocimientos científicos, ni a realizar prácticas, sino «hacer salir los conocimientos» de una manera participativa; hacerles ver cómo se estudiaba y cómo se resolvían las dificultades. Más que enseñar ciencia, Arcimís enseñaba a realizar procesos científicos —observaciones, mediciones, simulaciones, juegos - , y, para lograr estos propósitos, nada mejor que convertir la cátedra en un taller y a los alumnos en «expertos» astrónomos y predictores del tiempo. Su iniciativa de crear un competente aunque modesto observatorio que contaba con las secciones de astronomía y meteorología ${ }^{56}$, aparece en retrospectiva como una medida sumamente audaz ${ }^{57}$. Esa era probablemente la cualidad que Giner reclamaba a Arcimís para empresas de mayor vuelo pero para quien sus constantes alusiones a la creación de una cátedra de astronomía física resultaban casi irrealizables en el marco de una concepción social de la ciencia ${ }^{58}$.

55 Arcimís, A. Meteorología, Barcelona, Calpe. Se desconoce la fecha exacta de su publicación, pero existen por lo menos tres ediciones de la misma.

56 ARCimís, A. (1885), «El Observatorio de la Institución: Sección meteorológica», Boletín de la Institución Libre de Enseñanza, 9, 8-11, 28-30, 43-45; ARCIMís, A. (1885), «Sección astronómica», Boletín de la Institución Libre de Enseñanza, 9, 116-119.

57 Desde el Observatorio de la Institución, efectuó observaciones de eclipses y confeccionó una revista meteorológica mensual que se publicaba en el B.I.L.E. Véase, por ejemplo: ARCimís, A. (1884), «El eclipse de luna del 4 de octubre de 1884», Boletín de la Institución Libre de Enseñanza, 8, 310; ARCimís, A. (1885), «Revista meteorológica de los meses de enero, febrero, marzo y abril», Boletín de la Institución Libre de Enseñanza, 9, 57-59; 79-80; 108-111; 173-176.

58 La creación de la cátedra universitaria de Astronomía Física hubo de esperar hasta 1900, año en el que el Plan García Alix la incluyó en la sección de ciencias como curso de doctorado. El cargo fue desempeñado por el astrónomo Antonio Tarazona. Véase: TARAZONA, 
Durante el periodo de 1875 a 1887, Arcimís continuó una y otra vez proclamando el mismo lema regeneracionista que le había transmitido a Giner en sus primeras cartas: la consigna de que la clave para la regeneración astronómica y meteorológica de España se encontraba en Europa y de que la estrategia más adecuada para alcanzar dicho objetivo era la fundación de observatorios astrofísicos, servicios meteorológicos y redes de observadores aficionados. Mantuvo una convicción, casi pertinaz, en el valor de la ciencia como un instrumento imprescindible para la modernización del país. Entre las lagunas más significativas de la ciencia oficial, señalaba la falta de un centro astrofísico y de un servicio de predicción meteorológica, así como la ausencia de estudios sobre las nubes y los espectros estelares. Pero para Arcimís, el cultivo de las ciencias de observación era importante tanto para ensanchar el espíritu como para sacar beneficio de su utilidad. Para su proyecto renovador, Arcimís no se inspiró en la ciencia del Observatorio de Madrid ni en el de San Fernando, sino en el pragmatismo del servicio de predicción francés y en el buen hacer del Observatorio astrofísico de Berlín.

La fundación de un servicio meteorológico central era una necesidad acuciante. Sirva como dato que en Europa, a mediados de la década de los ochenta, solamente tres países, Grecia, Turquía y España, no disponían de un servicio central destinado a la previsión del tiempo y a la coordinación de redes meteorológicas locales ${ }^{59}$. Al igual que en la astronomía, los institucionistas se adhirieron a una regeneración meteorológica en la que Europa se vislumbraba como modelo. Se trataba especialmente de imitar la fundación de servicios de predicción meteorológica, análogos a los que existían en los países vecinos, que fueran capaces de anunciar, desde una perspectiva dinámica, las perturbaciones atmosféricas en un plazo de tiempo corto ${ }^{60}$. Giner, partidario de revoluciones silenciosas, consideraba que Arcimís, ardiente y de espíritu luchador, como él, era la mejor garantía para dirigir una Oficina de Meteorología.

A. (1901), Programa de las lecciones de Astronomía Física explicadas durante el curso de 1900 a 1901, Madrid, Est.Tip. «Sucesores de Rivadeneyra».

59 Para una visión global de la organización meteorológica europea a finales del siglo XIX, véase: HellmanN, G. (1881), «The Organization of the Meteorological Service in Some of the Principal Countries of Europe», Meteorological Magazine, 16, 33-39; 51-57; 69-73; 8389 ; 99-106; 12-128; 142-145; 158-161; 165-173.

60 Francia e Inglaterra sirvieron de modelos meteorológicos. Véase: DAVIS, J. (1984), «Weather Forecating and the Development of Meteorological Theory at the Paris Observatory, 1853-1878», Annals of Science, 41, 359-382, pp. 362-367; BURTON, J. (1986), «Robert Fitzroy and the Early History of the Meteorological Office», British Journal for the History of Science, 19, 147-176, pp. 150-152. 
Para entonces, Arcimís era considerado como uno de los líderes más capacitados de la generación de jóvenes institucionistas que se esforzaban por importar un nuevo profesionalismo científico en el que se subrayaba tanto el espíritu de investigación y observación como el servicio a la comunidad civil. A nivel científico y organizador, Arcimís se nutrió de los conocimientos prácticos que adquirió en sus viajes a Inglaterra y Francia en 1888, cuando fue recibido deferentemente por los directores de los Observatorios de Greenwich y de Kent y por el personal del Meteorological Office. Como parte de su talante europeo, promovió un internacionalismo fructífero, aprovechando las amistades que había cultivado años atrás y haciendo gala de un bagaje idiomático poco común. En busca de información para mejorar su plan de servicio meteorológico, escuchó de primera mano los consejos de meteorólogos de talla internacional, como eran R. H. Scout, director del Meteorological Office, E. Mascart, director del Bureau Central de Météorologie ${ }^{61}$, y A. Argot, distinguido climatólogo y autor del Traité Elémentaire de Météorologie, que adquiriría amplia difusión en España. Y lo que es más importante, la concepción de meteorología de Arcimís como un lazo entre el mundo científico y las demandas de los sectores agrícola y pesquero, que contaba con precedentes extranjeros, era sin embargo radical para los esquemas españoles de los años ochenta.

Con tales ideales, no es de extrañar que la creación del Instituto Central Meteorológico español, en 1887, fuera la consumación de sus reivindicaciones. En tal sentido, puede afirmarse que su apertura fue una conquista de la Institución Libre de Enseñanza. Pero durante los años de entresiglos, ésta fue una conquista a medias. El Servicio Meteorológico oficial fue el teatro de operaciones de un fenómeno de bipolaridad institucional, que se personificó en la rivalidad que mantuvieron el ICM y el Observatorio de Madrid, por una parte, y en el pulso político de sus respectivos valedores, por otra ${ }^{62}$. Al lado del ICM se posicionó el bando renovador, amparado por los liberales y los institucionistas, mientras que los Observatorios de Madrid y San Fernando contaron con el apoyo del bando continuista y oficial, sustentado por los conservadores. Lo que se debatió en la cámara del Congreso de los Diputados, en agrias sesiones sobre política científica, era el modelo más idóneo de instituir

61 Las teorías meteorológicas de Mascart estaban en línea con lo que Arcimís preconizaba. Véase: MASCART, E. (1881), La météorologie appliquée a la prévision du temps: leçon faite le 2 mars 1880 à l'École Supérieure de Télégraphie, Paris, Gauthier-Villars.

62 García de Pedraza, L.; GimÉnez de la CuAdra, J. M. (1985), Notas para la historia de la Meteorología en España, Madrid, Ministerio de Transportes, Turismo y Comunicaciones, pp. 40-41. 
la meteorología, el procedimiento más adecuado de organizar las actividades meteorológicas ${ }^{63}$.

\section{CONCLUSIONES}

En España, la recepción de la obra de Arcimís fue, con diferencia, menos favorable de lo que lo fue en Europa. Como se ha señalado, su posición de outsider de la astronomía española y su alineamiento con ideales institucionistas en pro de la regeneración y en contra de la ciencia oficial no contribuyeron a ello; e incluso, la controvertida creación — con posterior supresióndel Instituto Central Meteorológico, su testimonio en defensa de la libertad religiosa y, más concretamente, su decisiva participación en la difusión de la obra de Draper fueron juzgados con excesiva sospecha. Paradójicamente, fue entre los círculos astronómicos catalanes, además por supuesto de los ambientes institucionistas, donde las percepciones hacia su talante científico calaron más hondo. Su obra El Telescopio moderno, como hemos visto, había sido una referencia intelectual para los jóvenes astrónomos Comas y Fontserè 64 , mientras que éste último recibiría sus consejos y elogios cuando se hizo cargo del observatorio de la Academia de Ciencias de Barcelona en $1893^{65}$.

Durante el resto de su vida, Arcimís se mostró como una relevante figura pública al mando de un controvertido Servicio. Desde su influyente cargo de director del ICM, en su celo por difundir la utilidad de la meteorología, se embarcó en la publicación diaria de una modesta predicción del tiempo en el Boletín Meteorológico ${ }^{66}$ y en un sinfín de actividades divulgativas ${ }^{67}$. Su compromiso por los ideales liberales se mantuvo en pié pero, ahora, desde el púlpito de las instituciones oficiales. Poco a poco, la imagen del intrépido joven gaditano, lleno de excitantes proyectos, se transformó en la de un templado,

63 Anduaga, A. (2004), «Ciencia, ideología y política en España. Augusto Arcimís (1844-1910) y la creación del Instituto Central Meteorológico», Boletín dela Institución Libre de Enseñanza, 52, 95-112.

64 BARCA (1995), p. 798.

65 Fruto de esta amistad que se inició en 1892, ambos mantuvieron una correspondencia que se ha conservado. Véase: Cartoteca de Catalunya, Fons Eduard Fontserè, FF carp. 27, A. Arcimís.

66 El Boletín Meteorológico del ICM comenzó a editarse el 1 de marzo de 1893.

67 Véase, entre otros ejemplos, su participación en el Diccionario Enciclopédico hispanoamericano, Montaner y Simón, Barcelona, 1887-1910, 28 v.; y en el Almanaque de la Ilustración, Establ. Tip. de los sucesores de Rivadeneyra, Madrid, a partir de 1893. 
aunque reputado, sabio con fama de buen consejero. El relato de las peripecias de Arcimís a bordo del globo tripulado Urano, junto con el ingeniero militar Alfredo Kindelán, con motivo del eclipse de Sol de 1905, dan buena muestra de la vitalidad y la gallardía que aún atesoraba a la edad de sesenta años, lo cual le sirvió para lograr las primeras fotografías que se obtenían del anillo de Ulloa y del espectro de Brocker en esas circunstancias ${ }^{68}$.

A lo largo de toda su trayectoria, Arcimís fue condescendiente con las penurias económicas del país pero combatiente con el proceder de los hombres. Como un joven entusiasta seducido por las aspiraciones regeneracionistas de Giner, un agudo observador que había entablado una corriente de comunicación científica en el extranjero, y un hombre de su tiempo comprometido con las reivindicaciones liberales durante la turbulenta etapa de la Restauración borbónica, Arcimís estuvo a la cabeza de la regeneración científica e intelectual española. Como se ha intentado demostrar, esta batalla por la «europeización» de la astronomía y la meteorología españolas se sirvió de la combinación creativa entre sus experiencias en el extranjero y la delicada coyuntura social y económica de su propio país. En medio de la tensión generada entre una España aferrada al pasado y otra que deseaba participar en la construcción de una nueva sociedad, Arcimís ensalzó el papel de la historia en su juicio sobre la ciencia y en el ejercicio que debía hacerse de la misma.«Los hombres de ciencias, señalaba, descuidan bastante no ya la Historia política universal, sino también aquella parte que refiriéndose con especialidad al objeto de sus estudios, debía más poderosamente interesarles», en clara alusión a la historia de la ciencia $^{69}$. Aquí, tal vez mejor que en ningún otro lugar, se compendia su particular visión sobre las raíces de los males de la ciencia española.

68 Arcimís, A. (1906), «Nota del Instituto Central Meteorológico», en Eclipse total de Sol de 30 de agosto de 1905. Reseña de los trabajos efectuados para su observación, Madrid, Imprenta Instituto Geográfico y Estadístico, pp. 31-40.

69 La cita aparece en HERRERA RodríGUEZ, F. (1995), «Revistas gaditanas de la segunda mitad del siglo XIX», Llull, 18 (34), 105; extraída a su vez de ARCIMís, A. (1876), «Apuntes para la historia de la brújula», La Verdad, 54, p. 5; 57, p. 3. 